\title{
Understanding the implications of the future unmanned air traffic growth
}

\author{
Cristina Barrado*, Esther Salamí ${ }^{\dagger}$, Antonia Gallardo $\ddagger$, L. Xavier Herranz, Enric Pastor $₫$ \\ Computer Architecture Department \\ Technical University of Catalonia \\ Barcelona, Spain \\ *cristina.barrado@upc.edu, †esther.salami@upc.edu, †antonia.gallargo@upc.edu, 9 enric@ac.upc.edu
}

\begin{abstract}
In the next years, the unmanned air business is expected to have an average annual growth rate of 14.5 per cent. Last-mile delivery, inspection works and security tasks are the most expected missions that those unmanned aircraft (UA) will execute. Most of these missions are well suited for multi-copters: small airframes with vertical take-off and landing capabilities. Large fleets of UA will be managed by new aerial logistic centers where flight plans will be created and monitored, the payload will be prepared, and fast battery replacement will allow continuous flights to obtain maximum benefit. Beyond visual line-of-sight capabilities is a must for those logistic center businesses. Anticipating the scalability of unmanned aircraft growth is the aim of this paper. For this, a simulation tool has been developed which generates unmanned traffic flights from completely parameterized inputs: the geographic area and the type and number of operations, aircraft and operators. For this paper, the tested scenario is a logistics industrial polygon with increasing delivery traffic, the Martorell industrial area (5.8 $\mathbf{k m}^{2}$ ). Flights have a random altitude from $80 \mathrm{~m}$ to $120 \mathrm{~m}$. Enroute phases have some slight turns to make them more realistic. The time of departure follows a Box-Muller algorithm during the declared business hours, centered in the peak declared hour.

Index Terms-UA, Conflict distance, Capacity
\end{abstract}

\section{INTRODUCTION}

As the technology is reducing costs and sizes, the applications for building competitive market solutions based in unmanned aircraft are growing. Many companies, such as Volocopter, Lilium, Airbus, Pipstrel Ascendance and Wingcopter, are leading developments for cargo and passengercarrying vehicles [1].

The European drones outlook study [2] estimated that 400, 000 drones will be providing services in the European airspace by 2050, with a total market value of more than 10 billion Euros per year by 2035 and a compound annual growth rate of more than $11 \%$ during the $2020-2025$ period. In 2018, the FAA informed that in the US the number of registered drone operators reached the amount of one million [3] and growth perspectives are similar.

Most of this growth is today a reality for operations in notpopulated areas. Tasks such as electrical tower inspections, precise agriculture or wild life protection are being deployed worldwide. But the larger piece of the future cake is for the so called urban air mobility (UAM), being passenger transport and logistics of the last mile delivery the most common business cases.

In this paper, we address the scalability of the unmanned traffic for the delivery business case. Using as testbed an industrial area, Martorell, that has actual experience in a recurrent daily cargo delivery flight, we have studied the effects of extending the experience with the number of daily flights ranging from 36 to more than 9,000.

\section{PREVIOUS WORK}

A similar study has been conducted San Francisco Bay Area in the United States and the Norrkoping region in Sweden [4]. Direct flights are randomly generated in straight line from random locations. The probability for a landing spot is proportional to the inhabitants density. Potential conflict for different traffic density and for increasing conflict separation distances are studied. Special attention is given to the complexity of the conflicts, using as main metrics the clustered conflicts, this is, those conflicts that involve more than 2 aircraft. Nevertheless the paper does not consider the vertical dimension, assuming that the ground is flat and all the UA flight at the same altitude. These are very unrealistic assumptions.

Another interesting work on urban airspace traffic density estimation is presented in [5]. Authors found that the realistic scenario for 2035 in Paris indicates a traffic density of 87,260 parcel delivery drones within the urban airspace of Paris. In addition, the paper presents a cost-analysis study for fast-food delivery via drones relative to electric bikes.

The work in [6] seeks to assess the collision risk posed by an intruding UA within the airport-restricted terminal airspace, in the Singapore context, with minimal information on the UA, but based the characterization in the domains of ground, airspace and regulations in order to depict the unmanned operational environment above populated areas. Authors limited operations hours over certain areas. Urban area types like commercial, public, green urban areas and sport / leisure facilities are considered suitable for the deployment of drone operations at only at specific time frames.

The DACUS project [7] focuses on the balance between demmand and capacity balance of unmanned traffic in the urban environment. While large separation distances provide more safety, the implications on the capacity reduction, and 
thus in the satisfied demmand, are very relevant. Ongoing research is expected to provide suitable separation distances to support future and safe UAM business growth.

Other approaches focus on the difficulty of UA management. The work in [8] examines a lane-based airspace structure over city roads and roundabouts. The reasoning is that roads are public space, and people on the road would be protected within their cars should a UA fall to the ground. In [9], the authors proposed a new air traffic management method for UA based on global subdivision theory. They model four types of low-altitude air routes for $5 \mathrm{G}$ grids at national, regional, county and township level, which correspond to grid sizes of 1.85 $\mathrm{km}, 128 \mathrm{~m}, 64 \mathrm{~m}$ and $32 \mathrm{~m}$ respectivelly. The results of the simulated experiments indicated that, in the $5 \mathrm{G}$ environment, gridded airspace could effectively improve the efficiency of UAV trajectory planning and reduce the size of information storage in the airspace environment.

Finally, [10] outlines research toward a definition of well clear for small unmanned aircraft systems, based on airborne collision risk, for midterm concepts of operations at low altitudes in nonterminal airspace. The lack of small UA data and low altitude operations was addressed with extensive data collection and processing. The collision risk was found to not be sensitive to assumptions. This lack of sensitivity is attributed to generally slow small UA airspaces. Therefore, the UA Executive Committee (EXCOM) Science and Research Panel (SARP) recommends a simultaneously lost of horizontal separation of $2000 \mathrm{ft}$ and vertical separation of $250 \mathrm{ft}$ (600 $\mathrm{m}$ and $75 \mathrm{~m}$ respectivelly). Unlike the large UA well-clear definition and Traffic Alert and Collision Avoidance System (TCAS) alerting criteria, that adds a temporal component to the small UA well-clear recommendation, the authors do not consider it and propose a cylinder protection volume. In this work we will opt also for cyclider separation volumes, but of different sizes.

\section{CHARACTERIZATION OF THE DELIVERY OPERATIONS}

The CORINE Land Cover inventory differentiates 44 classes on land usage adding urban and rural, being Martorell classified as an industrial area with production activities.

Figure 1 shows the Martorell industrial area used for this study. Martorell is a city $30 \mathrm{~km} \mathrm{SW}$ from Barcelona and holds the SEAT headquarters and automobile factory, creating a large ecosystem area with third companies providing components to the factory. The area has a total of $5.8 \mathrm{~km}^{2}$ in a perimeter of $10 \mathrm{~km}$. Terrain is mainly flat but still ground elevation ranges from $135 \mathrm{~m}$ (South) to $220 \mathrm{~m}$ (West).

From 2018, Martorell factory is testing a daily delivery using a drone. It consists on a wheel component that uses a fixed route as a proof of concept of the capacities of a UA for industrial use.

For this study, we have considered a total of 4 drone operators sited close to the perimeter. All operators do businessto-business deliveries inside the polygon. They are fictitiously called Amazon, Wing, Correos, and Uber, and have a base fleet of $10,1,5$, and 2 drones respectively. The drone models are

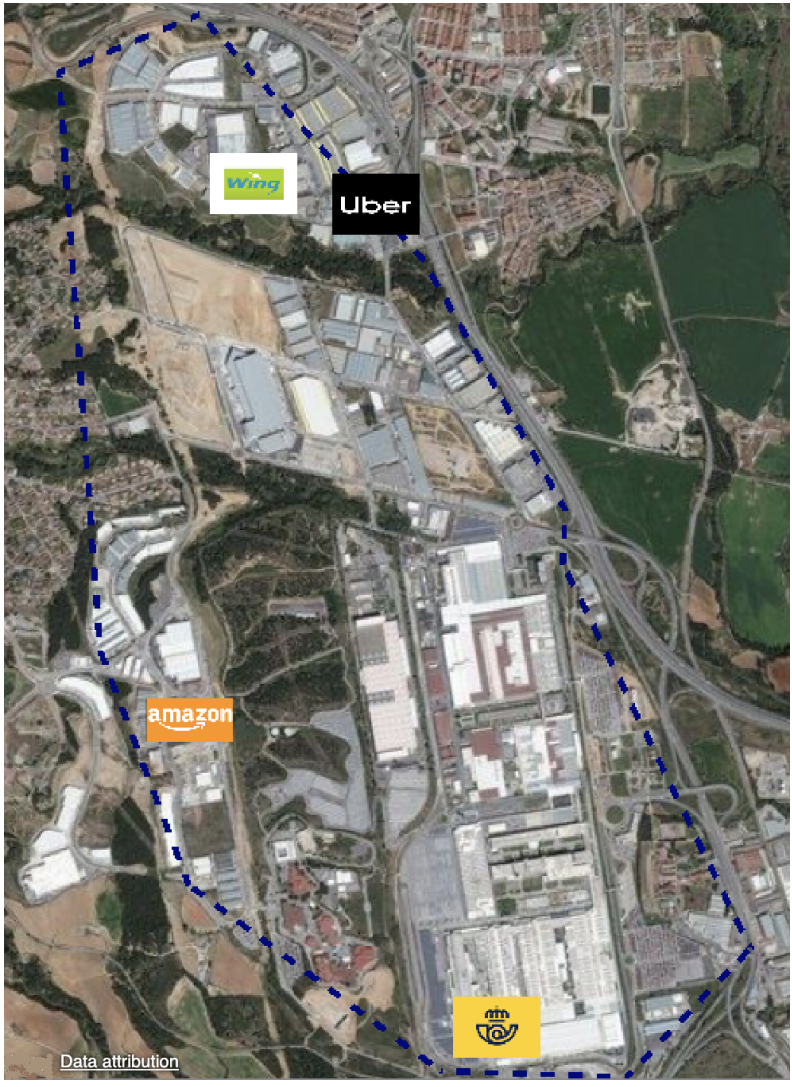

Fig. 1. Martorell industrial area and drone operators locations

randomly selected from a database containing common DJI, 3DR, and Parrot drone models.

Due to the terrain elevation, the drone operator vertiports are located at different altitudes (see Fig. 2). The airspace volume corresponding to a cylinder of radius $20 \mathrm{~m}$ and height $80 \mathrm{~m}$ surrounding each vertiport is considered managed by the corresponding operator. Cruise altitudes are randomly set from $80 \mathrm{~m}$ to $120 \mathrm{~m}$ above take-off, that is from $236.7 \mathrm{~m}$ to $327.8 \mathrm{~m}$ considering the different vertiport altitudes.

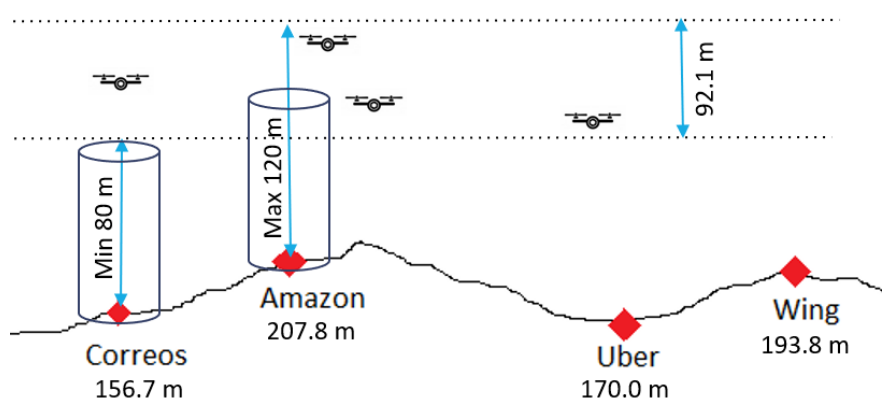

Fig. 2. Cruise flight altitudes

One drone can operate between 1 and 3 times per day. Each delivery operation consists on a vertical take-off to cruise 


\section{Horizontal profile}

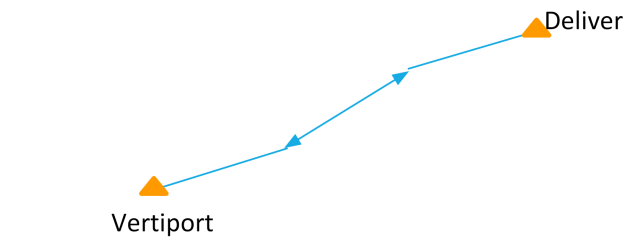

Vertical profile:

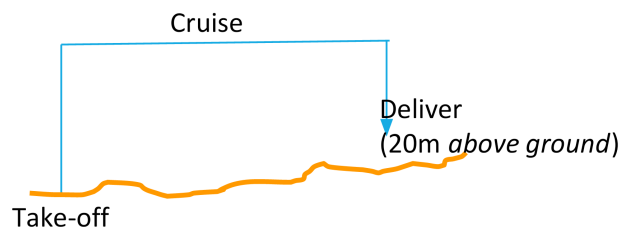

Fig. 3. Delivery flight profile

altitude, stable cruise flight to destination, vertical descend until $20 \mathrm{~m}$ over the terrain, hover for twenty seconds, and undo the same route to return. The cruise route consists on a random number of segments between 1 and 6 , random total distance of $4 \mathrm{~km}$ maximum, random direction from 1 to 360 degrees, and random intermediate angles from -15 to +15 degrees. Horizontal and vertical flight profiles are depicted in Fig. 3. For each operation, cruising speed, climbing speed, and descending speed are assigned randomly, considering the limitations of the drone model. Final speed values range from 4 to $25 \mathrm{~m} / \mathrm{s}$ and climb/descend from 3 to $10 \mathrm{~m} / \mathrm{s}$. Departure time follows a Box-Muller algorithm according to the working hours of the country. In the simulation set up, the working hours have been set to start at $7 \mathrm{am}$ and finish at $10 \mathrm{pm}$, with the peak business time at $12 \mathrm{pm}$.

\section{DELIVERY TRAFFiC AND CONFLICT DISTANCE PARAMETERS}

We have performed different simulations scaling the base configuration (described in Sec. III) by a traffic scale (TS) factor ranging from 1 to 256 . This results in traffic ranging from 36 to 9216 operations per day. Considering the area of the polygon, this means a traffic density between 6 and 1589 daily flights per $\mathrm{km}^{2}$.

Given the relative small area of Martorell, the average distance of each flight is $1.7 \mathrm{~km}$, with a average time of flight of 4 minutes and 40 seconds. An example of the flight routes generated in one simulation with $T S$ equal to 8 is shown in Fig. 4.

The simulator also analyzes the generated operations by searching the conflict situations created by them. Two aircraft are considered to be in conflict if the separation between them is less than a given value $H$ in horizontal and less than a

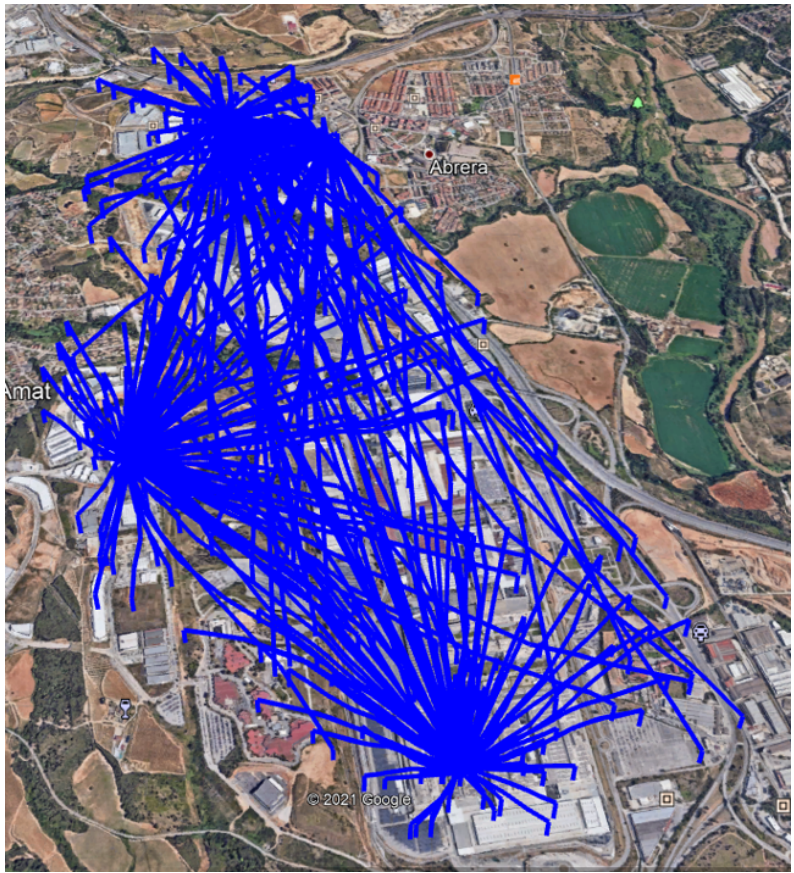

Fig. 4. Flight routes generated with traffic scale 8

value $V$ in vertical. In other words, we define the safe distance volume as a cylinder of radius $H$ and height $2 \mathrm{~V}$ centered in the aircraft. Then, two flights are in conflict if their safe distance volumes overlap. We have defined a base configuration with safe horizontal distance $H$ equal to $20 \mathrm{~m}$ and safe vertical distance $V$ equal to $5 \mathrm{~m}$. Different simulations have been performed scaling this base configuration by a factor of 1 , $2,4,8$, and 16. From now on, this scale factor is named safe distance scale $(D S)$. Figure 5 represents the safe distance volume at the different $D S$ values. Thus, our simulations consider separations from $20 \mathrm{~m}$ to $320 \mathrm{~m}$, being the vertical dimension always one fourth of the horizontal.

The proposed distances are all below the EXCOM SARP recommendations of $600 \mathrm{~m}$ horizontal and $80 \mathrm{~m}$ vertical. The reason is that we consider manned traffic to be forbidden in Martorell area. Also because the speeds of the UA proposed are also below the ones considered by the EXCOM SARP. Anyway, distance for safe separation of UA is still a subject of research [7].

\section{Simulation RESUltS}

This section presents the results obtained in the simulations carried out. All the combinations of the values of $T S$ and $D S$ are summarized in Table I. Observe that the number of flights per day range from 36 to 9,216 . These traffics represent daily densities from 6 to 1,589 aircraft per square kilometre. Notice that the density is the projection into two dimensions of the $92.1 \mathrm{~m}$ altitude airspace volume defined for Martorell.

The following sections give especial atention to several conflict metrics. The total number of conflicts is in average around ten thounsands, from which 5.8 thousands are en-route 


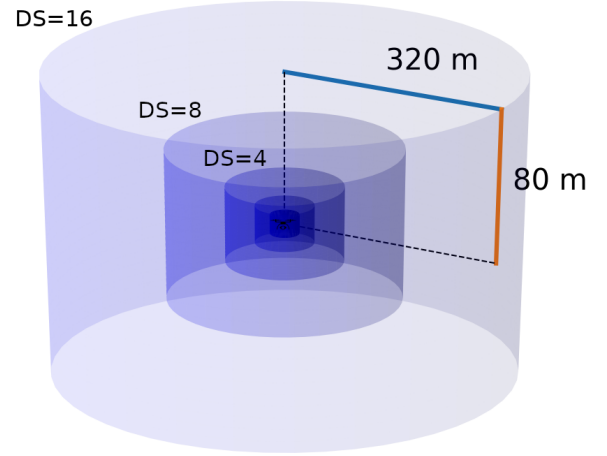

Fig. 5. Safe distance volume at different distance scales $(1,2,4,8,16)$

and the rest occur inside the vertiport airspace. For the less aggressive configurations the en-route conflicts represent only a $20 \%$ of the conflicts, while in the case of highly aggressive configurations their percent raises up to $65 \%$ of the total. For the rest of the paper we will present only en-route conflicts since it is assumed that conflicts inside vertiports are managed by the operator itself.

TABLE I

TRAFFIC SCALE AND SAFE DISTANCE SCALE CONFIGURATIONS

\begin{tabular}{|r|r|r|}
\hline Traffic scale (TS) & flights/day & (flights/day)/km ${ }^{2}$ \\
\hline 1 & 36 & 6 \\
2 & 72 & 12 \\
4 & 144 & 25 \\
8 & 288 & 50 \\
16 & 576 & 99 \\
32 & 1152 & 199 \\
64 & 2304 & 397 \\
128 & 4608 & 794 \\
256 & 9216 & 1589 \\
\hline Safe distance scale (DS) & Horizontal (m) & Vertical $(\mathbf{m})$ \\
\hline 1 & 20 & 5 \\
2 & 40 & 10 \\
4 & 80 & 20 \\
8 & 160 & 40 \\
16 & 320 & 80 \\
\hline
\end{tabular}

\section{A. Number of conflicts}

Figure 6 shows the overall number of conflicts occurred in the different configurations. We do not consider conflicts inside vertiports between flights of the vertiport operator, since it is assumed that they are managed by the operator itself. As expected, the number of conflicts increases exponentially with both traffic and safe distance. For $T S$ lower than 8 , the number of conflicts is practically zero, and they start to be significant after 16. For $T S$ equal to 8 (50 flights per day per $\mathrm{km}^{2}$ ), the number of conflicts per day varies from 6 (2\% the number of flights) to 89 (30\% the number of flights) depending on the safe distance scale, with a significant increase when $D S$ changes from 4 to 8 , where the number of conflicts increases from 19 (6\% the number of flights) to 60 (22\% the number of flights). For a given value of $D S$ equal to 4 , the number of conflicts noticeably worsens at $T S$ between 32 (460 conflicts) and 64 (1683 conflicts).
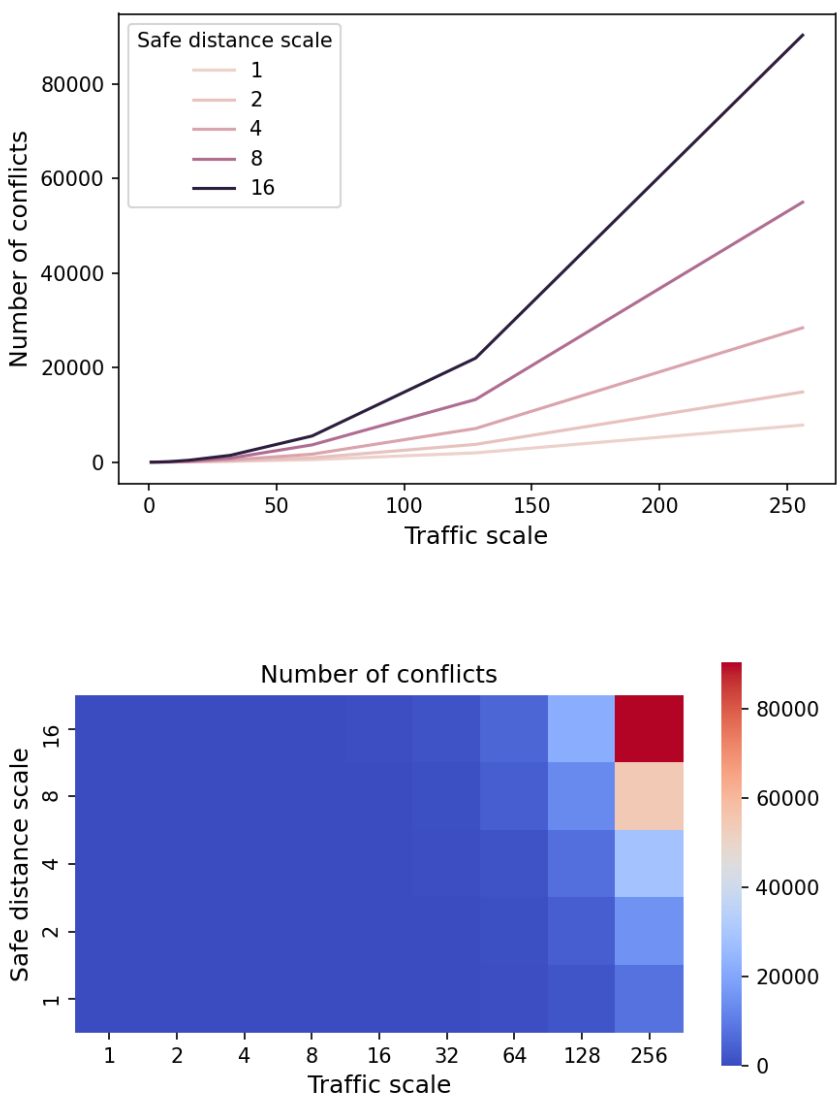

Fig. 6. Number of conflicts per day for different traffic scale (TS) and safe distance scale (DS) configurations

It is worth noting that the instantaneous traffic at the moment of the conflict, this is, number of actual aircraft enroute, is of 18 aircraft in average, with a maximum of 77 for the worst case. These numbers represent instantaneous densities of 3.1 and 13 aircraft $/ \mathrm{km}^{2}$ respectivelly, showing that there is still enogh free airspace for the deconflicting manoeuvers. Remember that a third dimension of more than $90 \mathrm{~m}$ is also available.

\section{B. Conflict clusters}

To assess the potential complexity of conflict resolution, we focus on the number of flights involved in spatial and temporal concurrent conflicts. Two conflicts are considered to be concurrent in space and time if they have one flight in common and both occur in a temporal window of 30 seconds (from 15 seconds before the closest point of approach to 15 seconds after it). Concurrent conflict clusters can be represented as graphs where the nodes are the flights and the edges represent the conflicts between those flights. Fig. 7 shows one cluster of size 6 and 10 conflicts. In this case, for example, flight ' $\mathrm{C}$ ' has conflicts with all the other flights in the cluster during the time window of $30 \mathrm{~s}$, while flight ' $\mathrm{A}$ ' 
has conflict only with 'B' and ' $\mathrm{C}$ '. The edges are annotated with the time of conflict, fixed as the time of the closest point of approach.

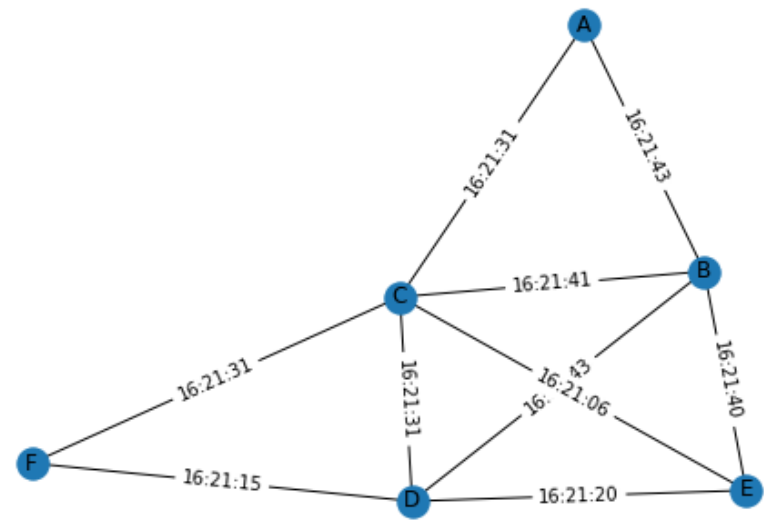

Fig. 7. Example of cluster with 6 flights (blue bullets) involved in conflicts and a total of 10 conflicts (lines between bullets).

Figure 8 shows the number of conflict clusters for the different configurations under study. We can see that the safe distance scale is a key parameter to determine the number of clusters. As the volume of the safe distance increases, so does the number of conflicts, and the conflicts merge into the same group, so we have fewer but much more complex groups.

Figure 9 shows the total number of conflicts and how many of them (in dark blue) are part of a cluster. Only the results for $D S$ equal to 4 and $T S$ from 16 to 128 are included for clearness. Concurrent conflicts become relevant for $T S$ between 32 and 64, with the percentage of conflicts belonging to a cluster being $40 \%$ and $60 \%$ respectively.

The complexity of the clusters is analyzed in Table II. It shows the cluster size, that is the number of flights (or nodes), and the number of conflicts (or edges) for different $T S$ and $D S$ values. Both, the mean and maximum value are included. Note that the simplest cluster would be made up of 3 flights and 2 conflicts. It can be seen that, in general, the clusters are small, with only 3 flights involved in concurrent conflicts. The mean value remains comparatively low even in the most aggressive configurations. However, the maximum value increases drastically for the last rows of the table. Worst cases are for $T S$ equal to 256 and $D S$ equal to 8 and 16, where the largest cluster contains $32 \%$ and $77 \%$ of the flights respectively. In all other cases, the cluster size is generally less than $1 \%$ of the traffic, and always less than $5 \%$.

The behavior is similar for the number of conflicts. It is worth noting that, in general, the number of conflicts is lower that the number of flights, meaning that not all flights are in conflict with each other. This is not true for the worst cases. For $T S$ equal to 256 and $D S$ equal to 16 , the number of conflicts in the biggest cluster is more than ten times the number of flights, showing that in average each aircraft is simultaneously in conflict with other 10 .

The distribution of the cluster size is better observed in Fig. 10. Note that the y-axis scale is logarithmic for clearness.
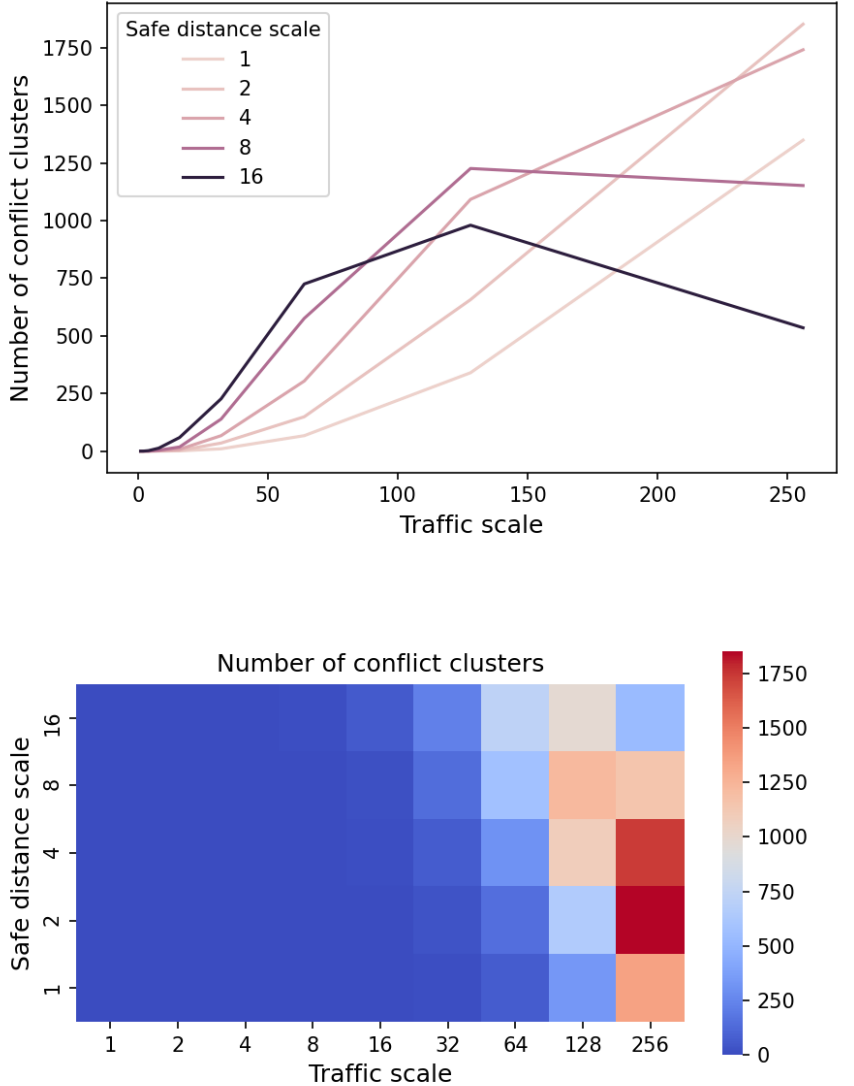

Fig. 8. Number of conflict clusters per day for different traffic scale (TS) and safe distance scale (DS) configurations

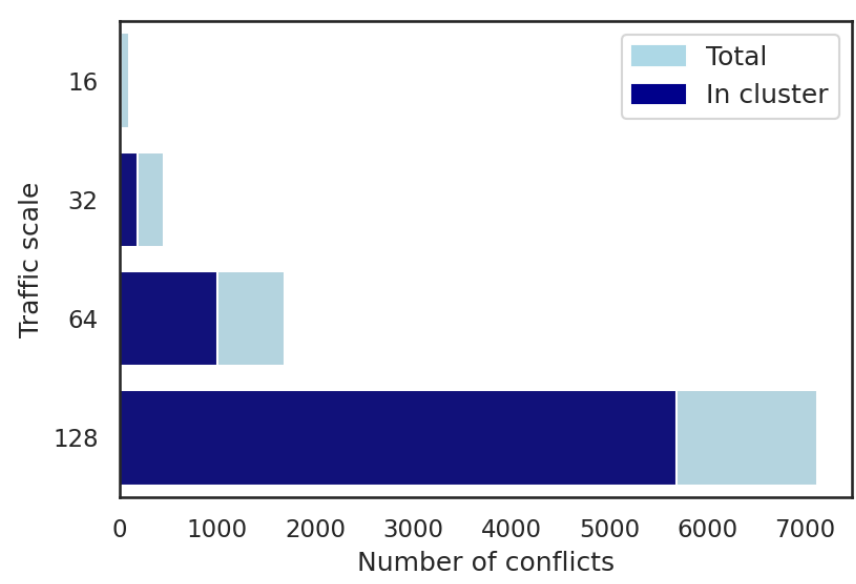

Fig. 9. Number of conflicts that belong to a cluster for safe distance scale (DS) equal to 4

Configurations with $T S$ below 16 are not included, as they are not relevant. The boxplot shows that the mean and standard deviation remain low for all the configurations, but outliers appear increasingly separated from the mean as we move to more aggressive configurations. 
TABLE II

CLUSTER SIZE AND NUMBER OF CONFLICTS FOR DIFFERENT TRAFFIC SCALE (TS) AND SAFE DISTANCE SCALE (DS) CONFIGURATIONS

\begin{tabular}{|r|c|c|c|c|c|}
\hline \multicolumn{7}{|c|}{ Number of flights per cluster (mean, max) } \\
\hline TS & DS=1 & DS=2 & DS=4 & DS=8 & DS=16 \\
\hline $\mathbf{1}$ & $(-,-)$ & $(-,-)$ & $(-,-)$ & $(-,-)$ & $(-,-)$ \\
$\mathbf{2}$ & $(-,-)$ & $(-,-)$ & $(-,-)$ & $(-,-)$ & $(-,-)$ \\
$\mathbf{4}$ & $--,-)$ & $(-,-)$ & $(-,-)$ & $(-,-)$ & $(3,3)$ \\
$\mathbf{8}$ & $(-,-)$ & $(3,3)$ & $(3,3)$ & $(3,3)$ & $(3,4)$ \\
$\mathbf{1 6}$ & $(4,4)$ & $(3,3)$ & $(3,3)$ & $(3,4)$ & $(4,7)$ \\
$\mathbf{3 2}$ & $(3,4)$ & $(3,5)$ & $(3,6)$ & $(3,10)$ & $(4,12)$ \\
$\mathbf{6 4}$ & $(3,7)$ & $(3,7)$ & $(4,9)$ & $(5,18)$ & $(5,32)$ \\
$\mathbf{1 2 8}$ & $(3,13)$ & $(4,14)$ & $(5,27)$ & $(7,121)$ & $(9,197)$ \\
$\mathbf{2 5 6}$ & $(4,21)$ & $(6,42)$ & $(9,195)$ & $(13,2963)$ & $(21,7098)$ \\
\hline \multicolumn{7}{|c|}{ Number of conflicts per cluster (mean, max) } \\
\hline TS & DS=1 & DS=2 & DS=4 & DS=8 & DS=16 \\
\hline $\mathbf{1}$ & $(-,-)$ & $(-,-)$ & $--,-)$ & $(-,-)$ & $(-,-)$ \\
$\mathbf{2}$ & $(-,-)$ & $(-,-)$ & $(-,-)$ & $(-,-)$ & $(-,-)$ \\
$\mathbf{4}$ & $(-,-)$ & $(-,-)$ & $(-,-)$ & $(-,-)$ & $(2,2)$ \\
$\mathbf{8}$ & $(-,-)$ & $(3,3)$ & $(3,3)$ & $(2,2)$ & $(2,3)$ \\
$\mathbf{1 6}$ & $(3,3)$ & $(2,2)$ & $(2,3)$ & $(2,5)$ & $(3,10)$ \\
$\mathbf{3 2}$ & $(2,3)$ & $(3,4)$ & $(3,8)$ & $(3,13)$ & $(4,27)$ \\
$\mathbf{6 4}$ & $(3,8)$ & $(3,12)$ & $(3,15)$ & $(5,38)$ & $(6,82)$ \\
$\mathbf{1 2 8}$ & $(3,13)$ & $(4,24)$ & $(5,56)$ & $(10,460)$ & $(22,1474)$ \\
$\mathbf{2 5 6}$ & $(4,31)$ & $(7,86)$ & $(15,645)$ & $(46,22898)$ & $(168,79602)$ \\
\hline
\end{tabular}
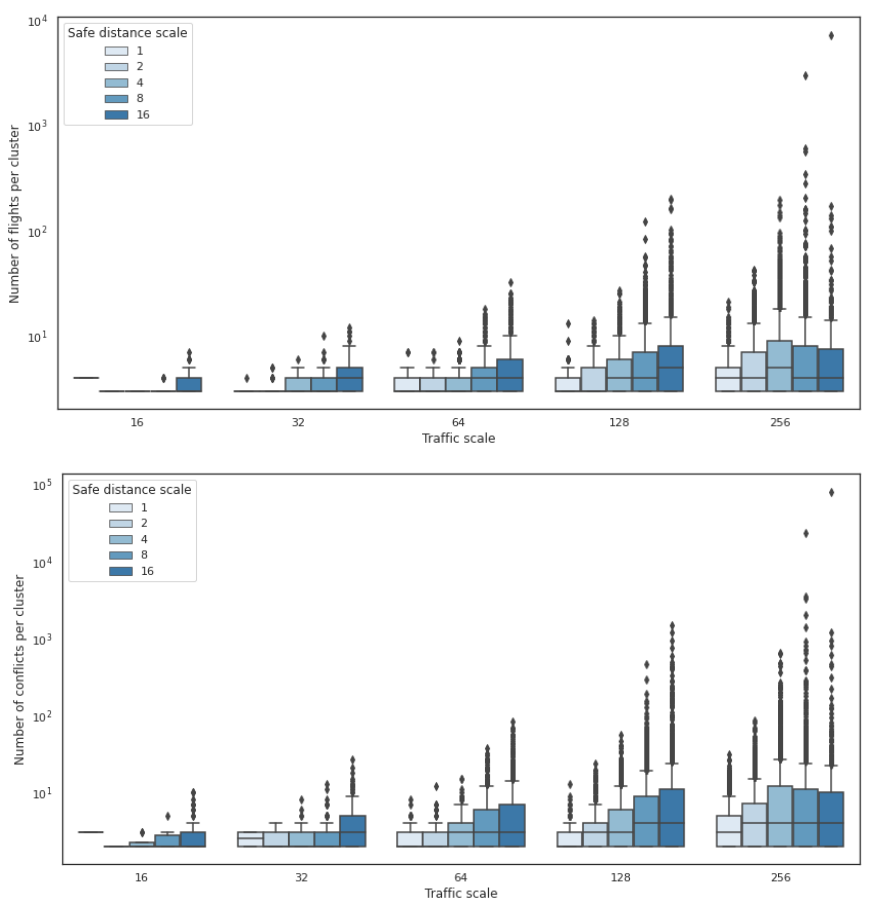

Fig. 10. Distribution of the number of flights and the number of conflicts in clusters for different traffic scale (TS) and safe distance scale (DS) configurations

\section{Time in conflict}

Table III shows the mean conflict duration and the maximum conflict duration in seconds. As it was expected, it depends mainly on the safe distance. The mean duration goes from around 4 seconds for the smallest safe distance volume to around 24 seconds for the largest one. The maximum time spent in one conflict is one minute. In our scenario, the routes are not very long and the average operating time is 4.7 minutes. Half a minute is not a long time, but it represents $10 \%$ of the average operating time.

TABLE III

TIME IN CONFLICT FOR DIFFERENT TRAFFIC SCALE (TS) AND SAFE DISTANCE SCALE (DS) CONFIGURATIONS

\begin{tabular}{|r|c|c|c|c|c|}
\hline \multicolumn{6}{|c|}{ Conflict duration in seconds (mean, max) } \\
\hline $\mathbf{T S}$ & DS=1 & $\mathbf{D S = 2}$ & $\mathbf{D S}=\mathbf{4}$ & $\mathbf{D S}=\mathbf{8}$ & $\mathbf{D S}=\mathbf{1 6}$ \\
\hline $\mathbf{1}$ & $(-,-)$ & $(-,-)$ & $(-,-)$ & $(-,-)$ & $(20,20)$ \\
$\mathbf{2}$ & $(-,-)$ & $(-,-)$ & $(21,33)$ & $(29,29)$ & $(20,30)$ \\
$\mathbf{4}$ & $(-,-)$ & $(2,4)$ & $(8,10)$ & $(15,30)$ & $(25,34)$ \\
$\mathbf{8}$ & $(5,25)$ & $(11,29)$ & $(13,33)$ & $(16,34)$ & $(22,34)$ \\
$\mathbf{1 6}$ & $(3,15)$ & $(6,30)$ & $(9,34)$ & $(15,34)$ & $(24,40)$ \\
$\mathbf{3 2}$ & $(4,26)$ & $(6,34)$ & $(9,34)$ & $(16,43)$ & $(24,44)$ \\
$\mathbf{6 4}$ & $(4,49)$ & $(6,34)$ & $(9,43)$ & $(15,35)$ & $(24,56)$ \\
$\mathbf{1 2 8}$ & $(4,34)$ & $(6,34)$ & $(9,42)$ & $(15,42)$ & $(24,56)$ \\
$\mathbf{2 5 6}$ & $(4,34)$ & $(6,39)$ & $(9,44)$ & $(15,58)$ & $(24,60)$ \\
\hline
\end{tabular}

More representative is the Normalized Time Spent in Conflict (NTSC). The NTSC is defined as the fraction of the operation time that the flight is in conflict. Figure 11 shows the average NTSC for the different configurations. It is calculated as the sum of the time that each flight is in conflict (with one or more flights) divided by the sum of the operation time of all the flights. Note that the time that the flight is in conflict does not have to be the sum of the duration of the conflicts, as the flight can conflict with more than one flight at a time.

We observe that the percentage of time spent in conflicts increases quickly with the safe distance. It is always less than $5 \%$ for the lowest DS configurations (DS $=1$ or 2 ), even in the case of higher traffic (TS = 256). For DS equal to 4,8 and 16, the NTSC reaches the 5\% at TS equal to 64,32 and 16 respectively.

\section{CONCLUSIONS}

To asses the potential scalability of unmanned aircraft traffic, we have simulated a scenario where 4 drone operators perform delivery operations inside a logistics industrial polygon with an area of $5.8 \mathrm{~km}^{2}$. Specifically, we have evaluated the number of conflicts, the complexity of the conflict clusters, and the time in conflicts for increasing delivery traffic and for different safe distances. We have observed that increasing the safe distance from $80 \mathrm{~m}$ to $160 \mathrm{~m}$ horizontally and from 20 $\mathrm{m}$ to $40 \mathrm{~m}$ vertically significantly limits the potential aircraft traffic. However, distance for safe separation of UA is still a subject of research and there is not a standard value.

For a safe distance volume of $80 \mathrm{~m}$ in horizontal and $20 \mathrm{~m}$ in vertical, which can be considered a fairly realistic value in the future, the capacity limit would be in the order of 1,000 - 2,000 flights per day (200 - 400 daily flights per $\mathrm{km}^{2}$ and within more than $90 \mathrm{~m}$ of altitude). With 2,000 flights, there were less than 1,700 conflicts in one day, with $60 \%$ of them overlapping in space and time, creating conflict clusters of 3 flights. Only 4 clusters were larger in size, with the largest one being a cluster of 9 flights. The mean duration of conflicts was 9 seconds. In average, this time represents the $4 \%$ of the overall operating time. Doubling the traffic (800 daily flights 

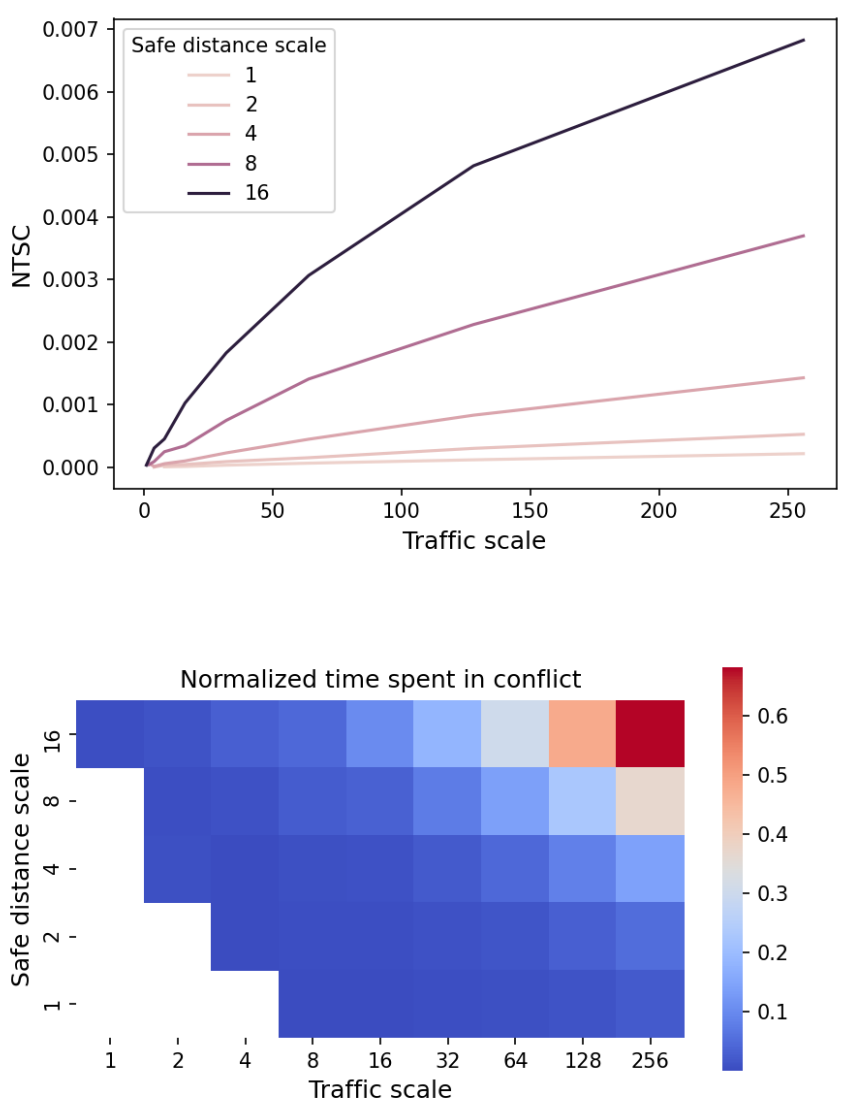

Fig. 11. Normalized time spent in conflict (NTSC) for different traffic scale (TS) and safe distance scale (DS) configurations

per $\mathrm{km}^{2}$ ) would increase the number of conflicts up to 7,000. Even though most conflicts continue to affect only two or three flights at a time, complex concurrent conflict clusters of up to 56 flights appear.

This study has focused on estimating the potential traffic capacity in a specific scenario. To establish stronger conclusions, it will be necessary to consider a greater diversity of parameters, such as the type of area, the number of operators or the existence of conflict resolution mechanisms. On the other hand, the developed framework is a good starting point to investigate new approaches in areas such as conflict detection and conflict resolution.

\section{ACKNOWLEDGMENT}

This work was funded by the Ministry of Economy, Industry, and Competitiveness of Spain under GrantNumber TRA2016-77012-R.

\section{REFERENCES}

[1] EASA, "Study on the societal acceptance of urban air mobility (uam) operations," 2021. [Online]. Available: https://www.easa.europa.eu/sites/default/files/dfu/uam-full-report.pdf

[2] S. J. Undertaking, "European drones outlook study. unlocking the value for europe," SESAR, Brussels, 2016.
[3] A. Boyd, "1 million drones, operators register to fly in us," 2018. [Online]. Available: http://www.nextgov.com/emerging-tech/2018/01/1million-drones-operators-register-fly-us/145440/

[4] V. Bulusu, R. Sengupta, V. Polishchuk, and L. Sedov, "Cooperative and non-cooperative uas traffic volumes," in 2017 International Conference on Unmanned Aircraft Systems (ICUAS). IEEE, 2017, pp. 1673-1681.

[5] J. Dunham, "Risk analysis framework for unmanned systems," Ph.D. dissertation, Georgia Institute of Technology, 2020.

[6] C. J. Wang, S. K. Tan, and K. H. Low, "Collision risk management for non-cooperative uas traffic in airport-restricted airspace with alert zones based on probabilistic conflict map," Transportation research part C: emerging technologies, vol. 109, pp. 19-39, 2019.

[7] D. Consortium, "Structures and rules in capacity constrained (urban) environment," 2021. [Online]. Available: https://dacusresearch.eu/reports-and-downloads/

[8] D. Sacharny and T. C. Henderson, "A lane-based approach for largescale strategic conflict management for uas service suppliers," in 2019 International Conference on Unmanned Aircraft Systems (ICUAS). IEEE, 2019, pp. 937-945.

[9] W. Zhai, B. Han, D. Li, J. Duan, and C. Cheng, "A low-altitude public air route network for uav management constructed by global subdivision grids," Plos one, vol. 16, no. 4, p. e0249680, 2021.

[10] A. Weinert, S. Campbell, A. Vela, D. Schuldt, and J. Kurucar, "Wellclear recommendation for small unmanned aircraft systems based on unmitigated collision risk," Journal of air transportation, vol. 26, no. 3, pp. 113-122, 2018. 